\title{
Frequency Analysis for Functionally Graded Material Cylindrical Shells: A Significant Case Study
}

\author{
Rabia Anwar (D), ${ }^{1}$ Madiha Ghamkhar, ${ }^{1}$ Muhammad Imran Khan, ${ }^{1}$ Rabia Safdar $\left(\mathbb{D},{ }^{2,3}\right.$ \\ Muhammad Zafar Iqbal, ${ }^{1}$ Wasim Jamshed, ${ }^{4}$ Esra Karatas Akgül $\mathbb{D},{ }^{5}$ and M. Prakash $\mathbb{D}^{6}$ \\ ${ }^{1}$ Department of Mathematics and Statistics, University of Agriculture, Faisalabad 38000, Pakistan \\ ${ }^{2}$ Department of Mathematics, College of Arts and Sciences, University of Jhang, Jhang 35200, Pakistan \\ ${ }^{3}$ Department of Mathematics, Lahore College Women University, Lahore 54000, Pakistan \\ ${ }^{4}$ Department of Mathematics, Capital University of Science and Technology, Islamabad 44000, Pakistan \\ ${ }^{5}$ Department of Mathematics, Art and Science Faculty, Siirt Üniversity, TR-56100 Siirt, Turkey \\ ${ }^{6}$ Department of Mathematics, Dr. N.G.P. Institute of Technology, Coimbatore, India
}

Correspondence should be addressed to Esra Karatas Akgül; esrakaratas@siirt.edu.tr and M. Prakash; drprakash@drngpit.ac.in

Received 7 August 2021; Revised 16 September 2021; Accepted 7 October 2021; Published 5 November 2021

Academic Editor: Rossana Dimitri

Copyright (C) 2021 Rabia Anwar et al. This is an open access article distributed under the Creative Commons Attribution License, which permits unrestricted use, distribution, and reproduction in any medium, provided the original work is properly cited.

Cylindrical shells play an important role for the construction of functionally graded materials (FGMs). Functionally graded materials are valuable in order to develop durable materials. They are made of two or more materials such as nickel, stainless steel, zirconia, and alumina. They are extremely beneficial for the manufacturing of structural elements. Functionally graded materials are broadly used in several fields such as chemistry, biomedicine, optics, and electronics. In the present research, vibrations of natural frequencies are investigated for different layered cylindrical shells, those constructed from FGMs. The behavior of shell vibration is based on different parameters of geometrical material. The problem of the shell is expressed from the constitutive relations of strain and stress with displacement, as well as it is adopted from Love's shell theory. Vibrations of natural frequencies (NFs) are calculated for simply supported-simply supported (SS-SS) and clamped-free (C-F) edge conditions. The Rayleigh-Ritz technique is employed to obtain the shell frequency equation. The shell equation is solved by MATLAB software.

\section{Introduction}

A shell is a structure consisting of curved sheets of material. A shell is the best effective way of consuming the material and can be very valuable in case of stowage of liquids and solids. Shells have different structures such as elliptic parboiled, hyperbolic parboiled, and circular cylindrical. Shell structures are generally used structural mechanisms in manufacturing designs. Vibration of cylindrical shells is a general field of research in mechanical dynamics. These shells are made from various kinds of materials. There are many types of shells, but CS is most important. The high strength, heat resistance, and corrosion resistance of FGM provide a reliable guarantee for the development of the nextgeneration nuclear industry. Nuclear power generation, nuclear power, and the storage of nuclear weapons are extremely dangerous. As a high-strength, heat-resistant, and shielding material, functionally graded materials show great superiority in the construction materials of nuclear furnaces and inner wall materials of nuclear furnaces, which greatly protect the safety of the nuclear industry.

Nowadays, FGMs are applicable in various fields including automotive, aerospace, mining, energy, and medical fields. Cylindrical shells are applicable in different appliances of mechanics, civil, nuclear, and chemical industries. They have different layers, e.g., single layer, bilayers, and trilayers. These layers are made up of "functionally graded" (FG) and "isotropic" materials [1]. Functionally graded materials are mostly utilized in the vast area of engineering and technology. Functionally graded material is a combination of more than one material. These materials are stainless steel (SS), zirconia ( $\mathrm{Zr})$, and nickel (Ni). The scientists from Japan 
(1884) purposed the group of materials with the conception of FGMs. These types of materials are manufactured by the combination of different materials employing the powder metallurgy method (powder metallurgy method is an industrial technique for attaining metals, such as powders). They contain vibration in essential fraction volume that takes to continual transform in the porosity and composition microstructure. These results are employed as a gradient in thermal and mechanical characteristics. Mostly, FGMs are applicable in the environment of maximum temperature. FGMs are used to construct the barrier of thermal materials. The analysis on FGMs tells that they are massive but mostly restrained to test the deformation and thermal stress [2]. The vibrational characteristics of the CS are composed of FGMs. There are multiple uses of FGMs in the industry as they are the most significant materials. They are auspicious materials and have the ability to continue their presence in a maximum produced temperature condition. An enormous amount of research work has been completed on the functionally graded CS. The vibration analysis depends on four clamped conditions employed on both shell ends. These conditions were expressed by Swaddiwudhipong et al. [3]. Rayleigh-Ritz method is an energy variation method that is used to find the natural frequency (NF). In 1909, Walther Ritz introduced this method. Rayleigh-Ritz method is also known as Galerkin's method. In other words, Rayleigh-Ritz technique is an integral approach method. It is valuable for resolving the problems of structural mechanics. It is also called the variation approach as given by Preumont [4]. Ng et al. [5] exhibited the construction for the dynamic solidness examination of the FG shell under the periodic axial loading. By the equation of motion, they produced the Mathieu-Hill equations and examined their stability by Bolotin's method. Rahimi et al. [6] examined the behavior of the vibrational characteristic of the CS with the supports of the ring placed at the centre of the shell. They utilized energy functional to get motion's equation. They considered the "functionally graded cylindrical shell" composed of the combination of ceramic and metal. Lee and Lu [7] examined the free vibration of cylindrical shells filled with liquid. They utilized "Donnell's shell theory" to analyze the vibrations of cylindrical shells and employed "Hamilton's principle" to obtain the function of shell dynamics. To modify the equations of differential into algebraic, "Galerkin's method" was used. Tang et al. [8] established the analytical technique for the free vibrational analysis of the cylindrical shell with arbitrary edge condition by reverberation-ray matrix's method. They utilized the Flugge thin theory to resolve the motion's equation and accomplished the exact solutions of travelling and standing waves from along the direction of axial and circumferential, respectively. They studied the influence of elastic restraints on the parameters of frequency. Qin et al. [9] scrutinized the free vibrational behavior of CSs with random edge conditions. For the calculation of "elastic strain energy," they utilized Sander's theory. Artificial springs were carried out on the ends of the shells to symbolize the arbitrary edge conditions. A united solution for three specific sorts of enlargement function was developed by using the technique of Rayleigh-Ritz. Wang et al. [10] presented the Jacobi-Ritz formulation to explore the free vibrations of different coupled doubly curved revolt shell structures with subjective edge conditions. They recognized the multisegment apportioning methodology and Flügge's shell theory to set up the theoretic model. By utilizing the Rayleigh-Ritz strategy, the natural frequencies and mode shapes of the coupled shell structures are chosen. Trabelsi et al. [11] inspected the behavior of thermal buckling of FG cylindrical shells and plates. The governing equations were expanded by considering the theory of first-order shear distortion. To solve the thermal buckling problem, fournode shell elements were assumed. The calculation of the censorious buckling temperature of structures under nonuniform temperature rise depends on Gaussian numerical combination. The impact of material composition, edge condition, thermal loading, and geometrical parameters of shells on the thermal buckling conduct of FGM structures was also examined. Daud and Viswanathan [12] introduced the vibrational conduct of the symmetric angle-ply layered circular cylindrical shell filled by calm fluid. The motion equation of the cylindrical shell as far as stress and moment resultants was obtained from the first-order shear distortion theory. To attain the differential equations with the rotational function and displacements, the relation of "straindisplacement and stress-strain" was assumed. The investigation of frequency parameters was examined utilizing the parameters the thickness and length ratio, angle-ply, properties of material, and the number of layers with various edge conditions. Sheng and Wang [13] established a theoretic model in the thermal atmosphere to examine the nonlinear vibration and dynamic stability of the stiffened FG cylindrical shell. For the modelling of the stiffened FG cylindrical shell, they utilized different techniques and theories such as Bolotin's technique, smearing stiffener approach, and von Karman nonlinear and first-order shear deformation theory. To achieve the differential equation, modal analysis and Galerkin's method were employed. The influence of the thermal atmosphere and material features on the dynamic constancy and temporary and crucial resonance responses was also observed. Strozzi and Pellicano [14] analyzed the nonlinear vibration of the FGM cylindrical shell. They applied Sanders-Koiter theory to determine the finite amplitude of vibration and deliberately supported the clamped and free boundary conditions. They carried out numerical analysis to depict the nonlinear response by considering a diversity of axisymmetric and asymmetric modes. Rawat et al. [15] exhibited the finite element method (FEM) for the vibration analysis of the circular shell. They employed various shell theories for FEM results to report in the literature. To investigate the computational efficacy, they used block "Lanczos and subspace iteration methods." They investigated model frequencies for various types of closures. Budak et al. [16] used numerical and experimental approaches to determine the dynamic characteristics of the isotropic cylindrical shell. They applied the finite element method with Femar software to accomplish numerical calculations. In order to observe the interferential pattern of the vibrational shell, they developed the stroboscopic holographic interferometry technique. Mat Daud and 
Viswanathan [12] presented the vibrational behavior of the symmetric angle-ply layered circular shell filled with quiescent fluid. They derived the equation of motion from the first-order shear deformation theory and stated the wave equation. They solved the eigenvalue problem for the frequency parameter of spline coefficient and examined the frequency parameter with different edge conditions. For further interests, the reader may read [17-21].

\section{Materials and Methods}

Figure 1 shows a cylinder-shaped shell in which $R$ is the radius, $h$ is the thickness, and $L$ is the length. The orthogonal coordinate system $(x, \theta, z)$ is established at the central surface of the CS, where $x, \theta$, and $z$ lie in the axial, circumferential, and radial directions of the shell, and $\left(u_{1}, v_{1}, w_{1}\right)$ are the displacements of the shell in $x, \theta$, and $z$ directions, respectively. The strain energy of a cylindrical shell is stated as

$$
M=\frac{1}{2} \int_{0}^{L} \int_{0}^{2 \pi}\{\epsilon\}^{T}[S]\{\epsilon\} R \mathrm{~d} \theta \mathrm{d} x,
$$

where

$$
\{\epsilon\}^{T}=\left\{e_{1}, e_{2}, \gamma, \kappa_{1}, \kappa_{2}, 2 \tau\right\}
$$

where $e_{1}, e_{2}, \kappa_{1}, \kappa_{2}$, and $2 \tau$ represent the strains and curvatures' reference surface relations, respectively. A variety of shell theories utilized are [17-20]. Love's shell theory is the first shell model, and other models were obtained from the Love shell theory by adding few terms.

According to Love's shell theory, the strain and curvature displacement relations can be defined as

$$
\begin{aligned}
e_{1} & =\frac{\partial u_{1}}{\partial x}, \\
e_{2} & =\frac{1}{R}\left(\frac{\partial v_{1}}{\partial \theta}+w\right), \\
\gamma & =\frac{\partial v_{1}}{\partial x}+\frac{1}{R} \frac{\partial u_{1}}{\partial \theta}, \\
\kappa_{1} & =-\frac{\partial^{2} w_{1}}{\partial x^{2}}, \\
\kappa_{2} & =-\frac{1}{R^{2}}\left(\frac{\partial^{2} w_{1}}{\partial \theta^{2}}-\frac{\partial v_{1}}{\partial \theta}\right), \\
\tau & =-\frac{1}{R}\left(\frac{\partial^{2} w_{1}}{\partial x \partial \theta}-\frac{\partial v_{1}}{\partial x}\right) .
\end{aligned}
$$

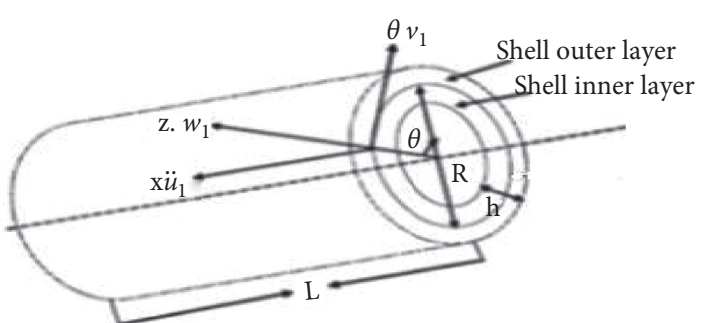

FIgURE 1: Geometry of the bilayer cylindrical shell.

$[S]$ is defined as

$$
[S]=\left[\begin{array}{cccccc}
A_{11} & A_{12} & 0 & B_{11} & B_{12} & 0 \\
A_{12} & A_{22} & 0 & B_{12} & B_{22} & 0 \\
0 & 0 & A_{66} & 0 & 0 & B_{66} \\
B_{11} & B_{12} & 0 & D_{11} & D_{12} & 0 \\
B_{12} & B_{22} & 0 & D_{12} & D_{22} & 0 \\
0 & 0 & B_{66} & 0 & 0 & D_{66}
\end{array}\right],
$$

where $[A]$ is extensional, $[B]$ is coupling, and $[D]$ is bending stiffness matrices $(i, j=1,2$, and 6$)$. They are defined as

$$
\left\{A_{i j}, B_{i j}, D_{i j}\right\}=\int_{-(h / 2)}^{h / 2} Q_{i j}\left(1, z, z^{2}\right) \mathrm{d} z .
$$

For isotropic materials, $Q_{i j}$ is the reduced stiffness stated as

$$
\begin{aligned}
Q_{11} & =Q_{22}=\frac{E}{1-v^{2}}, \\
Q_{12} & =\frac{v E}{1-v^{2}}, \\
Q_{66} & =\frac{E}{2(1+v)},
\end{aligned}
$$

where $E$ is Young's modulus and $v$ is Poisson's ratio. For the isotropic cylindrical shell, $B_{i j}$ (coupling stiffness) is zero, and for FGM cylindrical shells, $B_{i j}$ becomes nonzero. With the help of expressions (2) and (5), $M$ can be written as

$$
M=\frac{1}{2} \int_{0}^{L} \int_{0}^{2 \pi}\left\{\begin{array}{c}
A_{11} e_{1}^{2}+A_{22} e_{2}^{2}+2 A_{12} e_{1} e_{2}+A_{66} \gamma^{2}+2 B_{11} e_{1} \kappa_{1}+2 B_{12} e_{1} \kappa_{2}+2 B_{12} e_{2} \kappa_{1} \\
+2 B_{22} e_{2} \kappa_{2}+4 B_{66} \gamma \tau+D_{11} \kappa_{1}^{2}+D_{22} \kappa_{2}^{2}+2 D_{12} \kappa_{1} \kappa_{2}+4 D_{66} \tau^{2}
\end{array}\right\} R \mathrm{~d} \theta \mathrm{d} x
$$



form:

By putting (3) and (4) in (8), $M$ attains the following

$$
\begin{aligned}
& M= \frac{1}{2} \int_{0}^{L} \int_{0}^{2 \pi}\left\{A_{11}\left(\frac{\partial u_{1}}{\partial x}\right)^{2}+A_{22} \frac{1}{R^{2}}\right. \\
&\left(\frac{\partial v_{1}}{\partial \theta}+w\right)^{2}+2 A_{12} \frac{1}{R}\left(\frac{\partial u_{1}}{\partial x}\right)\left(\frac{\partial v_{1}}{\partial \theta}+w\right) \\
&+A_{66}\left(\frac{\partial v_{1}}{\partial x}+\frac{1}{R} \frac{\partial u_{1}}{\partial \theta}\right)^{2}-2 B_{11}\left(\frac{\partial u_{1}}{\partial x}\right)\left(\frac{\partial^{2} w_{1}}{\partial x^{2}}\right) \\
&-2 B_{12} \frac{1}{R^{2}}\left(\frac{\partial u_{1}}{\partial x}\right)\left(\frac{\partial^{2} w_{1}}{\partial \theta^{2}}-\frac{\partial v_{1}}{\partial \theta}\right) \\
&-2 B_{12} \frac{1}{R}\left(\frac{\partial v_{1}}{\partial \theta}+w_{1}\right)\left(\frac{\partial^{2} w_{1}}{\partial x^{2}}\right) \\
&-2 B_{22} \frac{1}{R^{3}}\left(\frac{\partial v_{1}}{\partial \theta}+w_{1}\right)\left(\frac{\partial^{2} w_{1}}{\partial \theta^{2}}-\frac{\partial v_{1}}{\partial \theta}\right) \\
&-4 B_{66} \frac{1}{R}\left(\frac{\partial v_{1}}{\partial x}+\frac{1}{R} \frac{\partial u_{1}}{\partial \theta}\right)\left(\frac{\partial^{2} w_{1}}{\partial x}-\frac{\partial v_{1}}{\partial x}\right)^{2} \\
&+D_{11}\left(\frac{\partial^{2} w_{1}}{\partial x^{2}}\right)^{2}+D_{22} \frac{1}{R^{4}}\left(\frac{\partial^{2} w_{1}}{\partial \theta^{2}}-\frac{\partial v_{1}}{\partial \theta}\right)^{2} \\
&+2 D_{12} \frac{1}{R^{2}}\left(\frac{\partial^{2} w_{1}}{\partial x^{2}}\right)\left(\frac{\partial^{2} w_{1}}{\partial \theta^{2}}-\frac{\partial v_{1}}{\partial \theta}\right) \\
&\left.+4 D_{66} \frac{1}{R^{2}}\left(\frac{\partial^{2} w_{1}}{\partial x \partial \theta}-\frac{\partial v_{1}}{\partial x}\right)^{2}\right\} R \mathrm{~d} \theta \mathrm{d} x \\
&
\end{aligned}
$$

The kinetic energy for a CS can be expressed as $K=\frac{1}{2} \int_{0}^{L} \int_{0}^{2 \pi} \rho_{T}\left\{\left(\frac{\partial u_{1}}{\partial t}\right)^{2}+\left(\frac{\partial v_{1}}{\partial t}\right)^{2}+\left(\frac{\partial w_{1}}{\partial t}\right)^{2}\right\} R \mathrm{~d} \theta \mathrm{d} x$, where $\rho_{T}$ is the mass density per unit length and is expressed as

$$
\rho_{T}=\int_{-(h / 2)}^{h / 2} \rho \mathrm{d} z .
$$

For a cylindrical shell, Lagrangian energy functional $\prod$ is stated as

$$
\Pi=K-M
$$

Axial modal dependence is employed to fulfill the edge conditions. For the approximation of axial modal dependence, different varieties of functions are adopted. The expression for axial, tangential, and radial displacements is supposed as

$$
\begin{aligned}
& u_{1}(x, \theta, t)=F \frac{\mathrm{d} \phi}{\mathrm{d} x} \cos n \theta \sin \omega \theta, \\
& v_{1}(x, \theta, t)=G \phi \sin n \theta \sin \omega \theta, \\
& w_{1}(x, \theta, t)=H \phi \cos n \theta \sin \omega \theta,
\end{aligned}
$$

where $F, G$, and $H$ are vibration amplitudes, $n$ is the circumferential wave number, and $\omega$ is the natural frequency (NF) of the shell. The axial function $\phi(x)$ is chosen to be a beam function as

$$
\begin{aligned}
\phi(x)= & \alpha_{1} \cosh \left(\frac{\lambda m x}{L}\right)+\alpha_{2} \cos \left(\frac{\lambda m x}{L}\right) \\
& +\varsigma_{m}\left(\alpha_{3} \sinh \left(\frac{\lambda m x}{L}\right)+\alpha_{3} \sin \left(\frac{\lambda m x}{L}\right)\right) .
\end{aligned}
$$

The original formula of the Lagrangian functional is established as

$$
\Pi=K_{\max }-M_{\max }
$$

where

$$
M_{\max }=\frac{\pi R}{2} \int_{0}^{L}\left[\begin{array}{c}
A_{11} F^{2}\left(\frac{d^{2} \phi}{d x^{2}}\right)^{2}+\frac{1}{R^{2}} A_{22}(n G+H)^{2} \phi^{2}+\frac{2}{R} A_{12}(n F G+F H) \phi \frac{d^{2} \phi}{d x^{2}} \\
+A_{66}\left(G-\frac{n F}{R}\right)^{2}\left(\frac{d \phi}{d x}\right)^{2}-2 B_{11} F H\left(\frac{d^{2} \phi}{d x^{2}}\right)^{2}+\frac{2}{R^{3}} B_{22}(n G+H)\left(n^{2} H+n F\right) \phi^{2} \\
+\frac{2}{R^{2}} B_{12}\left(n^{2} F H+n F G\right) \phi \frac{d^{2} \phi}{d x^{2}}-\frac{2}{R} B_{12}\left(n G H+H^{2}\right)+\phi \frac{d^{2} \phi}{d x^{2}}+\frac{4}{R} \bar{B}_{66}\left(G-\frac{n F}{R}\right) \\
(n H+G)\left(\frac{d \phi}{d x}\right)^{2}+D_{12} H^{2}\left(\frac{d^{2} \phi}{d x^{2}}\right)+\frac{1}{R^{2}} D_{22}\left(n^{2} H+n G\right)^{2} \phi^{2}-\frac{2}{R^{2}} D_{12}\left(n^{2} H^{2}+n G H\right) \\
\left.\phi \frac{d^{2} \phi}{d x^{2}} \frac{4}{R^{2}} D_{66}(n H+G)^{2}\left(\frac{d \phi}{d x}\right)^{2}\right] \mathrm{d} x .
\end{array}\right]
$$


To obtain the shell frequency equation, the energy functional is maximized with regard to the unknowns $F, G$, and $H$ of the CS. The following expression is attained as

$$
\frac{\partial \Pi}{\partial F}=\frac{\partial \Pi}{\partial G}=\frac{\partial \Pi}{\partial H}=0 .
$$

The shell frequency is expressed in the eigenform as

$$
\left[\begin{array}{lll}
C_{11} & C_{12} & C_{13} \\
C_{12} & C_{22} & C_{23} \\
C_{13} & C_{23} & C_{33}
\end{array}\right]\left[\begin{array}{l}
F \\
G \\
H
\end{array}\right]=\Omega\left[\begin{array}{ccc}
I_{2} & 0 & 0 \\
0 & I_{4} & 0 \\
0 & 0 & I_{4}
\end{array}\right]\left[\begin{array}{l}
F \\
G \\
H
\end{array}\right],
$$

where MATLAB software is used which is a dominant tool for the quick and converging solution of numerical problems. The MATLAB command, e.g., $(K, M)$, is utilized to extract the eigenvalues and eigenvectors in the procedure of shell natural frequencies and mode shapes, respectively.

2.1. Functionally Graded Materials (FGMs). Functionally graded materials are attained by mixing more than one material. Generally, FGMs are applied in the environment of maximum temperature. The material property $P$ is stated as a function of temperature $T$, where $T$ is expressed in kelvin [21].

$$
P=P_{0}\left(P_{-1} T^{-1}+1 P_{1} T+P_{2} T^{2}+P_{3} T^{3}\right),
$$

where $P_{0}, P_{-1}, P_{1}, P_{2}$, and $P_{3}$ are the coefficients of $T$.

The material property $P$ of FGM and the volume fraction of the composing materials are stated as

$$
P=\sum_{j=1}^{k} P_{j} V_{f j} .
$$

The summation of volume fraction of all composing materials must be unity.

$$
\sum_{j=1}^{k} V_{f j}=1 .
$$

The volume fraction for the single-layer CS having thickness $h$, made up of two composing materials $M_{1}$ (stainless steel) and $M_{2}$ (nickel), can be written as

$$
V_{f}=\left(\frac{z+0.5 h}{h}\right)^{N} \quad 0 \leq N \leq \infty,
$$

where $z$ is the thickness variable and $N$ is the power law exponent. $E$ is Young's modulus, $v$ is Poisson's ratio, and $\rho$ is the mass density, which can be stated for the FGM cylindrical shell consisting of two materials:

$$
\begin{gathered}
E=\left(E_{1}-E_{2}\right)\left(\frac{2 z+h}{2 h}\right)^{\mathrm{N}}+E_{2}, \\
v=\left(v_{1}-v_{2}\right)\left(\frac{2 z+h}{2 h}\right)^{N}+v_{2}, \\
\rho=\left(\rho_{1}-\rho_{2}\right)\left(\frac{2 z+h}{2 h}\right)^{N}+\rho_{2} .
\end{gathered}
$$

Volume fraction for the bilayer cylindrical shell is represented by the following three cases.

Case I:

When the inner layer is made of FGM and outer is of isotropic,

$$
V_{f}=\left(\frac{2 z}{h}+1\right)^{N} \quad 0 \leq N \leq \infty .
$$

Case II:

When the inner layer is of isotropic and outer is of FGM,

$$
V_{f}=\left(\frac{2 z}{h}\right)^{N} \quad 0 \leq N \leq \infty .
$$

Case III:

When both inner and outer layers are of FGM,

$$
\begin{aligned}
V_{f} & =\left(\frac{2 z}{h}+1\right)^{N}, \\
V_{f} & =\left(\frac{2 z}{h}\right)^{N}, \\
0 & \leq N \leq \infty .
\end{aligned}
$$

Young's modulus, Poisson's ratio, and mass density for the bilayer cylindrical shell can be stated as

$$
\begin{aligned}
& \stackrel{1}{E}_{F G M}=\left(E_{1}-E_{2}\right)\left(\frac{2 z}{h}+1\right)^{N}+E_{2}, \\
& \stackrel{2}{E}_{F G M}=\left(E_{1}-E_{2}\right)\left(\frac{2 z}{h}\right)^{N}+E_{2}, \\
& \stackrel{1}{v}_{F G M}=\left(v_{1}-v_{2}\right)\left(\frac{2 z}{h}+1\right)^{N}+v_{2}, \\
& \stackrel{2}{v}_{F G M}=\left(v_{1}-v_{2}\right)\left(\frac{2 z}{h}\right)^{N}+v_{2}, \\
& \stackrel{1}{\rho}_{F G M}=\left(\rho_{1}-\rho_{2}\right)\left(\frac{2 z}{h}+1\right)^{N}+\rho_{2}, \\
& \stackrel{2}{\rho}_{F G M}=\left(\rho_{1}-\rho_{2}\right)\left(\frac{2 z}{h}\right)^{N}+\rho_{2} .
\end{aligned}
$$

\section{Results and Discussion}

To justify the exactness and proficiency of the current results, simply supported-simply supported cylindrical shells are matched with $[22,23]$ (see Table 1) and [24] (see Table 2). Table 3 is compared with [2]. These comparisons show that the current outcomes are accurate, efficient, and valid. Ritz technique is utilized to accomplish the current result. This technique depends on the regulation of minimization of energy. 
TABLE 1: Comparison of frequency parameters (FPs) $\Omega=\omega R \sqrt{\left(1-v^{2}\right) \rho / E}$ for a SS-SS isotropic CS $(m=1, L=20, R=1, h=0.01$, and $v=0.3)$.

\begin{tabular}{lcc}
\hline$n$ & {$[23]$} & Current \\
\hline 1 & 0.016101 & 0.016101 \\
2 & 0.009382 & 0.009378 \\
3 & 0.022105 & 0.022102 \\
4 & 0.042095 & 0.042094 \\
5 & 0.068008 & 0.068007 \\
\hline
\end{tabular}

TABle 2: Comparison of NF (Hz) for the SS-SS isotropic CS $(m=1, L=20, R=1$, and $h=0.002)$.

\begin{tabular}{lcc}
\hline$N$ & {$[24]$} & Current \\
\hline 1 & 13.548 & 13.548 \\
2 & 4.592 & 4.591 \\
3 & 4.263 & 4.262 \\
4 & 7.225 & 7.224 \\
\hline
\end{tabular}

TABLe 3: Comparison of NF (Hz) for the SS-SS FGM cylindrical shell $(m=1, L=20, R=1$, and $h=0.002)$.

\begin{tabular}{ccccccccc}
\hline & & \multicolumn{2}{c}{ Current } & \multicolumn{3}{c}{ C2] } & \multicolumn{2}{c}{ Current } \\
$N$ & $N=1$ & $N=5$ & $N=1$ & $N=5$ & $N=1$ & $N=5$ & $N=1$ \\
\hline 1 & 13.211 & 12.998 & 13.211 & 12.997 & 13.3210 & 13.526 & 13.322 \\
2 & 4.480 & 4.4068 & 4.4746 & 4.4047 & 4.4511 & 4.5836 & 4.5142 \\
3 & 4.1569 & 4.0891 & 4.1355 & 4.0900 & 4.1827 & 4.2536 & 4.1892 & 4.5755 \\
4 & 7.0384 & 6.9251 & 6.9997 & 6.9326 & 7.0903 & 7.2085 & 7.0995 & 7.1788 \\
5 & 11.241 & 11.061 & 11.181 & 11.075 & 11.3293 & 11.516 & 11.342 & 11.468 \\
\hline
\end{tabular}

\section{CASE-1}

\section{TYPE-I}

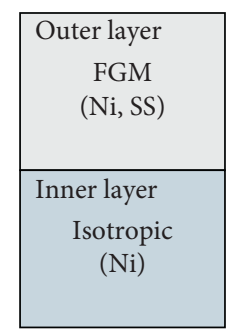

TYPE-III

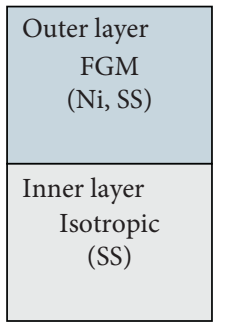

TYPE-II

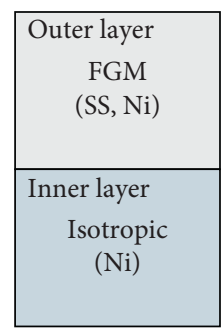

TYPE-IV

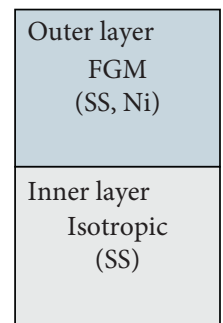

FIGURE 2: FGM type I and type II of the cylindrical shell.

In the functionally graded (FG) cylindrical shell, FGM is composed of stainless steel (SS) and nickel (see Figure 2). Two edge conditions are applied on it, which are given as simply supported-simply supported and clamped-free.

Table 4 represents the behavior of the NF (Hz) with $n$ for a type I and II functionally graded C-F and SS-SS cylindrical shell. Here, SPs are $m=2, L=20, N=1$, and $R=1$. In both types, natural frequency (NF) rises with an increase in $h$. The natural frequency increases for different ascending values of $h$. These values decrease from $n=1$ to 2 and then start to decrease with the increasing values of $n$. The influence of NFs in types I and II is the same.

The bilayer cylindrical shell is composed of two layers. In case I, the inner layer is made up of FGM, and the outer layer is of isotropic material. The FGM layer is made of nickel (Ni) and stainless steel (SS). In case II, both layers are composed 
TABle 4: Behavior of NF (Hz) with $n(m=1, N=1, L=100$, and $R=1)$.

\begin{tabular}{|c|c|c|c|c|c|c|c|c|}
\hline \multicolumn{9}{|c|}{ FG cylindrical shell } \\
\hline \multirow[b]{3}{*}{$N$} & \multicolumn{3}{|c|}{ Type I } & & \multicolumn{4}{|c|}{ Type II } \\
\hline & \multicolumn{2}{|c|}{$\mathrm{C}-\mathrm{F}$} & \multicolumn{2}{|c|}{ SS-SS } & \multicolumn{2}{|c|}{$\mathrm{C}-\mathrm{F}$} & \multicolumn{2}{|c|}{ SS-SS } \\
\hline & $h=0.1$ & $h=0.5$ & $h=0.1$ & $h=0.5$ & $h=0.1$ & $h=0.5$ & $h=0.1$ & $h=0.5$ \\
\hline 1 & 114.551 & 117.506 & 48.4992 & 49.1025 & 114.859 & 117.721 & 48.5028 & 49.0785 \\
\hline 2 & 98.5711 & 333.031 & 66.9496 & 315.299 & 99.1923 & 336.551 & 67.6230 & 318.867 \\
\hline 3 & 189.957 & 883.406 & 181.411 & 872.264 & 191.869 & 8933.21 & 183.357 & 882.139 \\
\hline 4 & 349.727 & 1660.62 & 346.023 & 1647.30 & 353.435 & 1679.25 & 349.747 & 1665.50 \\
\hline 5 & 560.698 & 2410.81 & 558.370 & 2407.14 & 566.702 & 2417.94 & 564.384 & 2414.93 \\
\hline 6 & 819.952 & 2893.14 & 818.135 & 2895.47 & 828.761 & 2901.69 & 826.952 & 2904.08 \\
\hline 7 & 1126.81 & 3375.37 & 1125.22 & 3378.12 & 1138.94 & 3385.35 & 1137.36 & 3388.12 \\
\hline 8 & 1481.07 & 3857.60 & 1479.60 & 3860.25 & 1497.03 & 3869.01 & 1495.56 & 3871.66 \\
\hline 9 & 1882.64 & 4339.82 & 1881.23 & 4342.29 & 1902.94 & 4352.65 & 1901.54 & 4355.12 \\
\hline 10 & 2331.47 & 4822.04 & 2330.10 & 4824.32 & 2356.64 & 4836.30 & 2355.27 & 4838.58 \\
\hline
\end{tabular}

TYPE-I

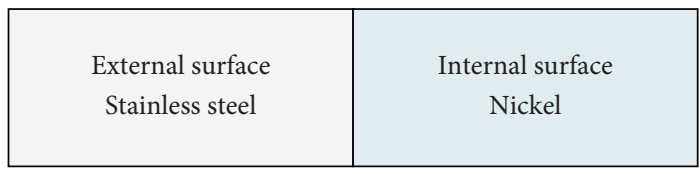

TYPE-II

\begin{tabular}{|c|c|}
\hline External surface & Internal surface \\
Nickel & Stainless steel \\
\hline
\end{tabular}

FIgURE 3: FGM cylindrical shell case I.

CASE-II

TYPE-I

TYPE-II
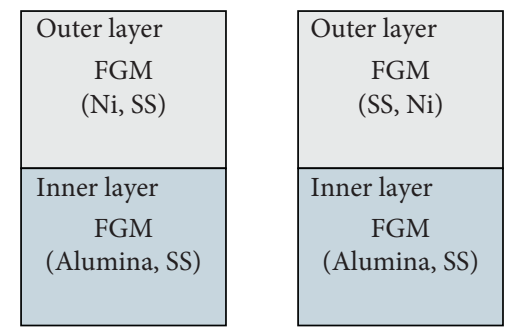

FIgURE 4: FGM cylindrical shell case II.

TABLE 5: Behavior of NF (Hz) with $n(m=1, h=0.1, N=1$, and $R=1)$.

\begin{tabular}{|c|c|c|c|c|c|c|c|c|}
\hline \multicolumn{9}{|c|}{ FG and isotropic bilayer cylindrical shell } \\
\hline \multirow[b]{3}{*}{$N$} & \multicolumn{3}{|c|}{ Type I } & & \multicolumn{4}{|c|}{ Type II } \\
\hline & \multicolumn{2}{|c|}{$\mathrm{C}-\mathrm{F}$} & \multicolumn{2}{|c|}{ SS-SS } & \multicolumn{2}{|c|}{$\mathrm{C}-\mathrm{F}$} & \multicolumn{2}{|c|}{ SS-SS } \\
\hline & $L=40$ & $L=60$ & $L=60$ & $L=80$ & $L=40$ & $L=60$ & $L=40$ & $L=60$ \\
\hline 1 & 29.1607 & 19.4438 & 1.48602 & 0.83728 & 29.3944 & 19.5996 & 3.36069 & 1.50074 \\
\hline 2 & 65.8666 & 64.2879 & 63.0416 & 63.0214 & 65.8841 & 64.2876 & 63.0840 & 63.0246 \\
\hline 3 & 178.846 & 178.450 & 178.186 & 178.163 & 178.808 & 178.410 & 178.209 & 178.144 \\
\hline 4 & 341.894 & 341.681 & 341.568 & 341.542 & 341.816 & 341.603 & 341.561 & 341.490 \\
\hline 5 & 552.552 & 552.388 & 552.316 & 552.290 & 552.427 & 552.264 & 552.266 & 552.192 \\
\hline
\end{tabular}


TABle 5: Continued.

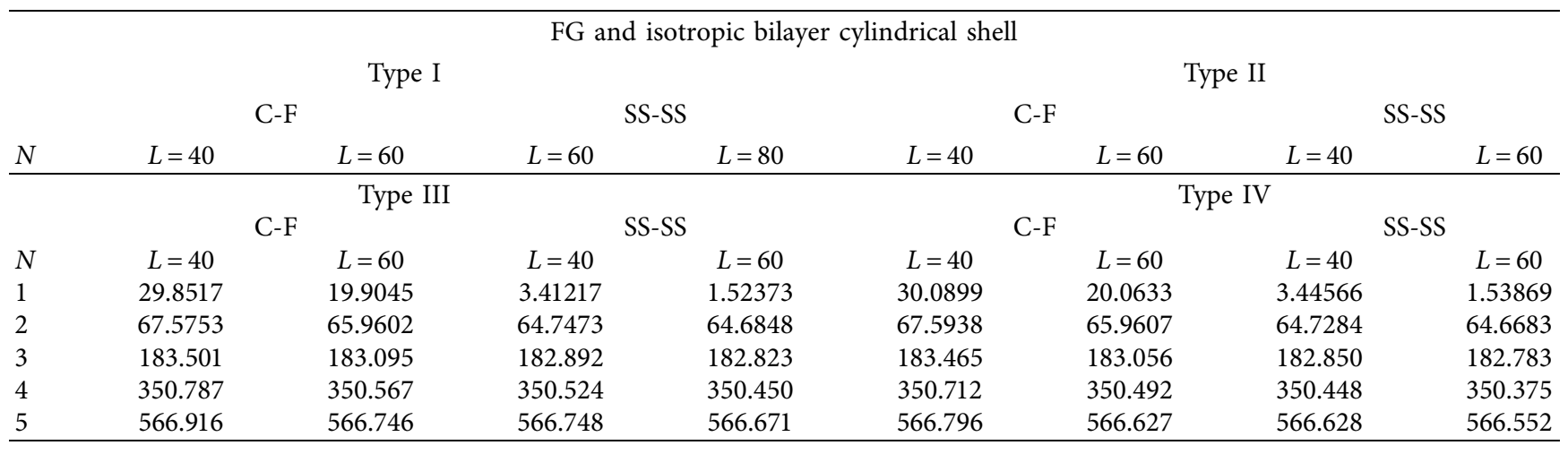

TABle 6: Behavior of NF (Hz) with $n(m=1, h=0.2, L=100$, and $R=1)$.

\begin{tabular}{|c|c|c|c|c|c|c|c|c|}
\hline \multicolumn{9}{|c|}{ FG bilayer cylindrical shell } \\
\hline \multirow[b]{3}{*}{$N$} & \multicolumn{3}{|c|}{ Type I } & & \multicolumn{4}{|c|}{ Type II } \\
\hline & \multicolumn{2}{|c|}{ C-F } & \multicolumn{2}{|c|}{ SS-SS } & \multicolumn{2}{|c|}{$\mathrm{C}-\mathrm{F}$} & \multicolumn{2}{|c|}{ SS-SS } \\
\hline & $N=6$ & $N=12$ & $N=6$ & $N=12$ & $N=6$ & $N=12$ & $N=6$ & $N=12$ \\
\hline 1 & 24.3969 & 309.190 & 1.11827 & 1.41774 & 24.2473 & 30.7109 & 1.11218 & 1.40889 \\
\hline 2 & 141.887 & 138.307 & 141.001 & 136.874 & 139.381 & 135.502 & 138.492 & 134.062 \\
\hline 3 & 398.705 & 387.262 & 398.516 & 386.976 & 391.617 & 379.314 & 391.429 & 379.027 \\
\hline 4 & 763.947 & 741.974 & 763.864 & 741.862 & 750.364 & 726.736 & 750.282 & 726.625 \\
\hline 5 & 1235.14 & 1199.70 & 1235.09 & 1199.64 & 1213.19 & 1175.06 & 1213.13 & 1174.99 \\
\hline 6 & 1811.63 & 1759.78 & 1811.59 & 1759.73 & 1779.43 & 1723.64 & 1779.39 & 1723.59 \\
\hline 7 & 2493.15 & 2421.98 & 2493.11 & 2421.94 & 2448.83 & 2372.24 & 2448.80 & 2372.20 \\
\hline 8 & 3279.54 & 3186.18 & 3279.50 & 3186.15 & 3221.26 & 3120.75 & 3221.22 & 3120.72 \\
\hline 9 & 4170.70 & 4052.34 & 4170.66 & 4052.30 & 4096.59 & 3969.12 & 4096.56 & 3969.09 \\
\hline 10 & 5166.52 & 5020.40 & 5166.48 & 5020.36 & 5074.73 & 4917.31 & 5074.70 & 4917.27 \\
\hline
\end{tabular}

of functionally graded material. In FGM, one layer is made up of nickel (Ni) and stainless steel (SS), while the other is of alumina (Al) and stainless steel (SS) (see Figures 3 and 4).

Table 5 shows the behavior of the NF $(\mathrm{Hz})$ with $n$ for type-I, II, III, and IV functionally graded and isotropic bilayer cylindrical shells with both edge conditions (C-F and SS-SS). The NF decreases when the value of $L$ rises. The natural frequency (NF) obtained from different values of $L$ increases when the circumferential wave number $n$ rises in all four types.

Table 6 represents the behavior of the NF (Hz) with $n$ for a type I and II FG bilayer CS. The NF rises when the value of power exponent $N$ increases. The NF obtained from different values of power exponent $N$ rises when $n$ increases in both types.

\section{Conclusion}

In this work, vibration analysis of natural frequencies is made for the bilayered cylindrical shell. The shell layers are constructed from functionally graded materials (FGMs). Different FGMs are used to compose the bilayered cylindrical shell such as SS, Ni, and Al. The present analysis is performed under different boundary conditions. The shell vibration is based on different parameters of geometrical material. The Lagrangian functional in the Rayleigh-Ritz technique has been used to solve the problem. In the current case, the axial mode is examined with the help of the characteristic beam function. Vibration of NFs is calculated for simply supported-simply supported and clamped-free edge conditions. Love's theory and Rayleigh-Ritz technique are applied to obtain the shell frequency equation. However, natural frequencies are examined with the effect of the volume fraction law. It is noticed that natural frequencies become high with the increase in thickness-to-radius ratios. The converse outcome is obtained with the increase of $L / R$ ratios. Natural frequencies are increased with the increase in power law exponent $N$.

MATLAB software has been used for the simplification of the equation of eigenvalues. The current study can be prolonged to analyze the cylindrical shell (CS) submerged in the fluid and multilayered FGM cylindrical shell.

\section{Abbreviations}

CS:

FGM:

SS-SS:

C-F:

NF:

E:

$N$ :
Cylindrical shell

Functionally graded material

Simply supported-simply supported Clamped-free

Natural frequency

Young's modulus

Power law exponent 


$\begin{array}{ll}\Pi: & \text { Lagrangian energy functional } \\ \text { SPs: } & \text { Shell parameters } \\ \rho: & \text { Mass density } \\ R: & \text { Radius } \\ h: & \text { Thickness } \\ n, m: & \text { Wavenumbers } \\ \Omega: & \text { Frequency parameters } \\ K: & \text { Kinetic energy } \\ \mathrm{Ni}: & \text { Nickel } \\ \mathrm{SS}: & \text { Stainless steel } \\ \mathrm{Al}: & \text { Alumina } \\ \mathrm{Zr}: & \text { Zirconia } \\ A_{i j}: & \text { Extensional } \\ B_{i j}: & \text { Coupling } \\ D_{i j}: & \text { Bending stiffness } \\ \nu: & \text { Poisson's ratio } \\ L: & \text { Length } \\ \{e\}: & \text { Strain vector } \\ \{\sigma\}: & \text { Stress vector } \\ \mathrm{Zr}: & \text { Zirconia } \\ M: & \text { Strain energy } \\ {[Q]:} & \text { Reduced stiffness matrix } \\ \phi(x): & \text { Axial function } \\ M_{x}, M_{\theta}, M_{x \theta}: & \text { Moment resultants. } \\ & \end{array}$

\section{Data Availability}

No data were used to support this study.

\section{Conflicts of Interest}

The authors declare that they have no conflicts of interest.

\section{Authors' Contributions}

R. Anwar provided the methodology, investigated the study, and reviewed and edited the article. M. Ghamkhar supervised, investigated, and conceptualized the study, provided the methodology, and edited the article. M. Imran and M. Zafar supervised, investigated, and conceptualized the study, provided the methodology, and wrote the article. R. Safdar, W. Jamshed, M. Prakash, and E. K. Akgül provided software, validated the study, and reviewed and edited the article.

\section{Acknowledgments}

This work was supported by the University of Agriculture Faisalabad, Pakistan.

\section{References}

[1] S.-R. Li, X.-H. Fu, and R. C. Batra, "Free vibration of threelayer circular cylindrical shells with functionally graded middle layer," Mechanics Research Communications, vol. 37, no. 6, pp. 577-580, 2010.

[2] C. T. Loy, K. Y. Lam, and J. N. Reddy, "Vibration of functionally graded cylindrical shells," International Journal of Mechanical Sciences, vol. 41, no. 3, pp. 309-324, 1999.
[3] S. Swaddiwudhipong, J. Tian, and C. M. Wang, "Vibrations of cylindrical shells with intermediate supports," Journal of Sound and Vibration, vol. 187, no. 1, pp. 69-93, 1995.

[4] A. Preumont, Twelve Lectures on Structural Dynamics, Springer, Berlin, Germany, 2013.

[5] T. Y. Ng, K. Y. Lam, K. M. Liew, and J. N. Reddy, "Dynamic stability analysis of functionally graded cylindrical shells under periodic axial loading," International Journal of Solids and Structures, vol. 38, no. 8, pp. 1295-1309, 2001.

[6] G. H. Rahimi, R. Ansari, and M. Hemmatnezhad, "Vibration of functionally graded cylindrical shells with ring support," Scientia Iranica, vol. 18, no. 6, pp. 1313-1320, 2011.

[7] L. T. Lee and J. C. Lu, "Free vibration of cylindrical shells filled with liquid," Computers \& Structures, vol. 54, no. 5, pp. 997-1001, 1995.

[8] D. Tang, G. Wu, X. Yao, and C. Wang, "Free vibration analysis of circular cylindrical shells with arbitrary boundary conditions by the method of reverberation-ray matrix," Shock and Vibration, vol. 2016, Article ID 3814693, 18 pages, 2016.

[9] Z. Qin, F. Chu, and J. Zu, "Free vibrations of cylindrical shells with arbitrary boundary conditions: a comparison study," International Journal of Mechanical Sciences, vol. 133, pp. 91-99, 2017.

[10] Q. Wang, K. Choe, D. Shi, and K. Sin, "Vibration analysis of the coupled doubly-curved revolution shell structures by using Jacobi-Ritz method," International Journal of Mechanical Sciences, vol. 135, pp. 517-531, 2018.

[11] S. Trabelsi, A. Frikha, S. Zghal, and F. Dammak, "A modified FSDT-based four nodes finite shell element for thermal buckling analysis of functionally graded plates and cylindrical shells," Engineering Structures, vol. 178, pp. 444-459, 2019.

[12] N. I. Mat Daud and K. K. Viswanathan, "Vibration of symmetrically layered angle-ply cylindrical shells filled with fluid," PLoS One, vol. 14, no. 7, Article ID e0219089, 2019.

[13] G. G. Sheng and X. Wang, "The dynamic stability and nonlinear vibration analysis of stiffened functionally graded cylindrical shells," Applied Mathematical Modeling, vol. 56, pp. 389-403, 2018.

[14] M. Strozzi and F. Pellicano, "Nonlinear vibrations of functionally graded cylindrical shells," Thin-Walled Structures, vol. 67, pp. 63-77, 2013.

[15] A. Rawat, V. A. Matsagar, and A. K. Nagpal, "Free vibration analysis of thin circular cylindrical shell with closure using finite element method," International Journal of Steel Structures, vol. 20, no. 1, pp. 175-193, 2020.

[16] V. D. Budak, A. Y. Grigorenko, M. Y. Borisenko, and E. V. Boichuk, "Natural frequencies and modes of noncircular cylindrical shells with variable thickness," International Applied Mechanics, vol. 2, no. 53, pp. 164-172, 2017.

[17] A. E. H. Love, "The small free vibrations and deformation of a thin elastic shell," Proceedings of the Royal Society of London, vol. 43 , no. $258-265$, pp. $352-353,1888$.

[18] J. L. Sanders Jr, An Improved First-Approximation Theory for Thin Shells, NASA, Washington, D.C., USA, 1959.

[19] W. Flügge, Statik Und Dynamik Der Schalen, Springer-Verlag, Berlin, Germany, 2013.

[20] V. Z. Vlasov, General Theory of Shells and Its Application in Engineering, NASA, Washington, D.C., USA, 1964.

[21] Y. Touloukian and C. Ho, "Properties of aluminum and aluminum alloys," in Thermophysical and Electronic Properties Information Analysis Center, 1973.

[22] C. T. Loy, K. Y. Lam, and C. Shu, "Analysis of cylindrical shells using generalized differential quadrature," Shock and Vibration, vol. 4, no. 3, pp. 193-198, 1997. 
[23] C. T. Loy and K. Y. Lam, "Vibration of cylindrical shells with ring support," International Journal of Mechanical Sciences, vol. 39, no. 4, pp. 455-471, 1997.

[24] M. N. Naeem and C. B. Sharma, "Prediction of natural frequencies for thin circular cylindrical shells," Proceedings of the Institution of Mechanical Engineers-Part C: Journal of Mechanical Engineering Science, vol. 214, no. 10, pp. 1313-1328, 2000. 